\title{
Diacronie
}

Studi di Storia Contemporanea

$N^{\circ} 22,2$ | 2015

Costruire

Michele Toss, Il Popolo Re. La canzone sociale a Parigi (1830-1848)

\section{Emilio Franzina}

\section{Q OpenEdition \\ Journals}

\section{Edizione digitale}

URL: http://journals.openedition.org/diacronie/2123

DOI: $10.4000 /$ diacronie.2123

ISSN: 2038-0925

\section{Editore}

Association culturelle Diacronie

\section{Notizia bibliografica digitale}

Emilio Franzina, « Michele Toss, II Popolo Re. La canzone sociale a Parigi (1830-1848) », Diacronie [Online], $N^{\circ} 22$, 2 | 2015, documento 21, online dal 01 juin 2015, consultato il 09 octobre 2020. URL : http:// journals.openedition.org/diacronie/2123; DOl : https://doi.org/10.4000/diacronie.2123 


\section{Diacronie}

N. 22 | 2|2015 Costruire. Rappresentazioni, relazioni, comunità

21/

\section{RECENSIONE:}

\section{Michele TOSS, Il Popolo Re. La canzone sociale a Parigi (1830-1848), Bologna, Clueb, 2014, 218 pp.}

a cura di Emilio FRANZINA *

Non sono poche le questioni su cui un'aneddotica attualizzante induce a soffermarsi quando, occupandosi di musica e storia, s'intenda tener conto, ad esempio, del successo planetario arriso negli ultimi anni, più che alla sua parabola alquanto complessa, a Bella Ciao, vero emblema canoro della Resistenza italiana e punta di diamante di un "italian combat folk for the masses" fatto proprio, grazie a una fortunata reinterpretazione dei Modena City Ramblers, appunto da masse imponenti di persone nelle più diverse parti del mondo. Ma sono, a ben vedere, per quanto assai distanti nel tempo o per genesi e modalità di diffusione dell'oggetto comune di studio, quasi le stesse questioni che in parte innervano la trama del bel libro imbastito a proposito del canto sociale a Parigi fra il 1830 e il 1848 da Michele Toss.

Un libro, sia detto in partenza, che si inserisce con punte di vera originalità nel dibattito in corso da lungo tempo, sia in Francia che in Italia, sull'uso delle fonti canore nella ricostruzione del passato a ridosso di importanti avvenimenti e di non meno rilevanti processi di trasformazione, anche politica, dell'esistente ${ }^{1}$. Il fatto di doversi misurare con tanti punti e spunti offerti dalla nuova storia sociale e culturale della musica e dei suoni che costituirono non tanto lo sfondo, quanto uno dei presupposti attivi, dalla Restaurazione in avanti, dei principali cambiamenti verificatisi in seno alle classi popolari a Parigi (e in misura più ridotta anche in altre città francesi: si pensi alla Nizza del giovane Garibaldi e alla sua nota passione per le canzoni di Béranger) spianando così la strada ai rivolgimenti e ai tentativi rivoluzionari del '48 europeo, costringe l'autore a un duplice sforzo: da un lato quello di confrontarsi assiduamente

${ }^{1}$ DARRIULAT, Philippe, La Muse du peuple. Chansons politiques et sociales en France 18151871, Paris, Presses Universitaires de Rennes, 2010. 
con gli esiti di molte ricerche altrui (in Italia di Leydi, Bermani, Jona, Pivato ecc., in Francia di Rancière, Darriulat, Millot ecc.) e da un altro quello, non meno impegnativo, di dotare di un corredo di documentazioni nuove e tutte di prima mano l'attenta ricostruzione del percorso compiuto dalla "parola operaia", ovvero dai punti vista espressi, con la voce e in musica, dai ceti artigiani e dalla stessa piccola borghesia urbana fra il 1830 e il 1848 .

Il discorso di Toss, che fa leva qui sui frutti di un paziente scavo archivistico condotto tra le fonti di polizia parigine (rapporti, relazioni prefettizie, ordinanze, ma anche testi, canzonieri ed opuscoli a stampa recuperati o individuati per la prima volta), prende le mosse da una realtà cittadina in grande fermento e da un paesaggio sonoro di transizione che nella musicalità diffusa degli anni seguiti alla caduta di Napoleone sembra ripigliare sulle prime i caratteri della sociabilità preindustriale con una folla vagante di colporteurs e di cantanti da strada, di saltimbanchi e di suonatori d'organetto o di altri strumenti (alcuni dei quali, sia detto en passant, provenienti da diverse zone dell'alta Italia). Essi, marcando una profonda differenza rispetto ad epoche successive e, dopo la metà del secolo XIX, anche rispetto alle opportunità di riproduzione e di trasmissione meccanica dei suoni che ci sono oggi più familiari, vengono ritratti dall'autore mentre si diramano quasi senza interruzione per le vie e per le piazze della capitale francese rivitalizzando una dimensione pubblica e collettiva del canto non ancora precisamente orientata verso le pur incipienti rivendicazioni sociali e come in bilico fra le eredità comportamentali di vecchio regime e la nostalgia in chiave patriottica per la figura e il mito dell'Imperatore. Sin dentro ai primi tempi della Monarchia di luglio, come minimo, trovano quindi spazio, oltre a ciò, le inevitabili riprese dell'innodia legata ai fasti della grande rivoluzione dell'89 (basti solo pensare alla lunga vita, qui e in ulteriori contesti, della Marsigliese a cui Toss dedica ovviamente un discreto spazio). Esse sono spesso certificate dall'enfatizzazione consapevole di motivi considerati adesso tout court sovversivi come la Carmagnole o il Chant du Départ. Anche i cantori plebei e gli esercenti di mestieri ambulanti che nei loro spostamenti e nel loro assiduo girovagare fra guinguettes e osterie, luoghi principi di ritrovo dei popolani e dei lavoratori manuali, continuano a privilegiare repertori ludici e d'intrattenimento spesso di contenuto irriverente e licenzioso, avvicinandosi la svolta del 1830 quando il loro numero raggiunge l'acme (quasi un migliaio quelli censiti dalla Prefettura di Parigi), segnalano tuttavia, con le stesse loro peripezie determinate dai controlli e dagli interventi censori o repressivi della gendarmeria e delle autorità, la ragguardevole metamorfosi del canto popolare che è in atto e che porterà nel volgere di breve tempo all'affermarsi di pratiche sociali assai incisive nella produzione e nel 
consumo di testi ad alto tasso rivendicativo. L'opera dei cantastorie e degli chansonniers des rues, spesso appoggiata ai contrafacta di motivi già noti a una variegata platea di operai e artigiani, innesca infatti, man mano, ma con efficacia propagandistica crescente e nettamente più elevata di quella dei pamphlets o delle brochures d'ispirazione repubblicana (frutto peraltro copioso dell'attività di molti crieurs de papiers publics), una spirale di suoni e di canti, creati e soprattutto gestiti dalla gente dei quartieri, che è destinata a ingigantirsi sino a configurare un intero e nuovo universo linguistico.

Il protagonismo popolare nell'opera di creazione e di promozione di una infinità di motivi inseguiti da Toss con acribia e in modo sistematico (sono centinaia i titoli e le citazioni testuali portate a sostegno) dimostra come non sia casuale l'inclinazione a innestare sul tronco originario del repubblicanesimo le ragioni di una protesta proletaria che progressivamente dilaga anche in rapporto al modificarsi delle condizioni di vita e di lavoro delle classi subalterne, intraviste e catalogate con viva inquietudine, ed anzi con preoccupazione evidente dopo l'avvento della monarchia orleanista, sotto specie di classi pericolose. Ma se questo è ciò che consta, dai lavori di Louis Chevalier in avanti ${ }^{2}$, a una storiografia sociale e politica agguerrita e bene informata, ciò che risalta nella lettura di Toss è il ruolo nevralgico e a lungo negletto della sfera emozionale nel garantire tra i componenti delle classi lavoratrici urbane, proprio attraverso il canto, la presa e la tenuta di quei sensi di solidarietà e di comune appartenenza che si convertiranno a tempo debito, ossia nel 1848, in precise richieste di giustizia sociale e di diritti validi per tutti i cittadini.

Dagli ambienti di lavoro a quelli dello svago, dagli atelier ai caffè, dai cabaret agli spacci di vino o alle gargotte (bettole, osterie, taverne ecc.) in cui non mancano da parte dei soggetti proletari i tentativi sintomatici d'imitazione delle esperienze borghesi di socializzazione comme il faut, se non proprio tutto, molto passa ed avviene al suono e al canto individuale $o$, più spesso, corale di canzoni che marcano altresì l'incedere di fenomeni quali la lettura collettiva dei giornali o l'associazionismo operaio sino a sfociare infine nella nascita e nella inarrestabile proliferazione delle goguettes divenute quasi da subito sinonimo di unione coreutica e musicale d’indubitabile valenza politica. Come luoghi privilegiati della sociabilità popolare inutilmente avversati dal potere monarchico orleanista, le goguettes parigine occupano la scena nonché la parte centrale e più densa del libro di Toss che si muove con abilità nel loro «intricato e

${ }^{2}$ CHEVALIER, Louis, La formation de la population parisienne au XIXe siècle, Paris, Presses Unversitaires de France, 1950; ID., Classi lavoratrici e classi pericolose: Parigi nella rivoluzione industriale, Bari, Laterza, 1976. 
complesso dedalo»3. In esso si mescolano fra danze, banchetti e pretesti conviviali forme le più diverse di aggregazione in fieri debitrici ora al mutualismo e a vecchi assetti corporativi, ora ai cerimoniali massonici e al più collaudato associazionismo del caveau o del ritrovo borghese già ben studiato da Maurice Agulhon e da Maria Malatesta 4 , ma ora e più spesso anche «ai riti e ai culti carnevaleschi e alla tradizione comica popolare»5. Ampliando a dismisura le funzioni e il concetto stesso di unione categoriale del compagnonnage o delle vecchie guinguettes quale riflesso di una più vasta rete di relazioni e di comunicazioni sociali in via di costituzione, le goguettes, dopo un avvio tollerato nell'età della Restaurazione, fra il 1830 e la vigilia della rivoluzione quarantottesca diventano segno e tramite a propria volta, ma "dal basso" stavolta, di una precoce modernizzazione di Parigi e senz'altro della polarizzazione, persino spaziale nel suo perimetro urbano, di rispettivi ambiti d'insistenza, uno borghese - quello dei nuovi strati sociali favoriti da Luigi Filippo, Roi citoyen, e dunque favorevoli alle sue esortazioni all'arricchimento privato - e uno operaio e artigiano, quello proletario incrementato dall'espansione manifatturiera di una vera miriade di aziende e d'imprese ancorché, nella media, di piccole o piccolissime dimensioni.

La mobilità necessitata e i frequenti spostamenti di sede delle goguettes per sfuggire ai controlli della polizia (per cui esse erano costrette talora ad abbandonare le zone centrali per le periferie come accadde più volte a quelle animate da uno chansonnier battagliero della fama di Charles Gille) anziché contenerli, esaltavano e dilatavano gli spazi pubblici democratici in cui si veniva sviluppando, sempre più all'insegna e con l'ausilio del canto, il discorso - giusta la definizione e le analisi di Andrea Lanza ${ }^{6}$ - «socialista fraternitario» ovvero la "presa di parola” degli operai. E ciò avveniva in luoghi d'incontro nei quali non a caso, in congiunture e frangenti di particolare tensione, sarebbero state erette di tempo in tempo, nel 1832, nel 1834, nel 1839 sino al 1848, alcune famose barricate e in cui ancora più spesso vennero intonate cruciali canzoni di lotta, specchio veridico dall'alterità sociale, culturale e politica di

\footnotetext{
3 TOSS, Michele, La canzone sociale a Parigi (1830-1848), Bologna, Clueb, 2014, p. 66.

4 AGULHON, Maurice, La sociabilité est-elle un object d'histoire? in ETIENNE, François (sous la direction de), Sociabilité et société bourgeoise en France, en Allemagne et en Suisse (17501850), Paris, Recherches sur les civilisations, 1986, pp. 13-25; ID., Histoire vagabonde. Ethnologie et politique dans la France contemporaine, Paris, Gallimard, 1988; MALATESTA, Maria (a cura di), Il salotto, il circolo e il caffè. I luoghi della sociabilità nella Francia borghese (1810-1848), Roma, Donzelli, 1993. Cfr. anche MAIULLARI, Maria Teresa (a cura di), Storiografia francese e italiana a confronto sul fenomeno associativo durante il $18^{\circ}$ e $19^{\circ}$ secolo (Atti delle giornate di studio: Torino, 6 e 7 maggio 1988), Torino, Fondazione Luigi Einaudi, 1990.

5 TOSS, Michele, op. cit., p. 66.

${ }^{6}$ LANZA, Andrea, L'abolizione del proletariato! Il discorso socialista fraternario. Parigi 18391847, Milano, Franco Angeli, 2010.
} 
buona parte della popolazione lavoratrice parigina. Che alcuni di quei luoghi coincidessero grosso modo con quelli fatiscenti e degradati poi scelti da Sue o da Balzac per descrivere la miserabilità dei quartieri più poveri di Parigi a confronto con la ricchezza e il dinamismo concettuale delle serate musicali e danzanti del "basso popolo" in marcia verso un'assunzione di coscienza e di sensibilizzazione politica ancora forse qua e là confusa, ma prefigurante gli sbocchi del '48, non deve scandalizzare o stupire. Grazie al canto engagée che sfruttava il piacere della compagnia, delle libagioni e persino del ballo si faceva strada l'idea repubblicana e democratica di un futuro migliore e proteso verso l'emancipazione sociale senza più remore né timori di ritorsioni se poteva succedere, ormai ben dentro agli anni Quaranta, che alcuni versi di una lettera in musica di Charles Gille à un fonctionnaire public à propos de la fermeture d'une société de chant si spingessero ad ammonire, evocando gli abusi perpetrati dalla censura a danno dei giornali, che «Le travailleur ne peut s'instruire/A leurs quotidiennes leçons/Mais il chante sans savoir lire/ Monseigneur, prends garde aux chansons». La goguette fondata da Gille e sciolta d'imperio dalla polizia, la Ménagerie, era la stessa, sia detto di passata, in cui il segnale d'apertura dei canti suonava: «les chansons politiques sont permises, on peut dire merde au roi»7.

La risata liberatoria e la beffa plebea, in realtà, riuscivano felicemente a convivere, nelle goguettes, con iniziative di beneficenza assai concrete e tese a rinsaldare l'uso di alcune pratiche di solidarietà in favore di vedove o di compagni rimasti senza lavoro e inoltre non stridevano affatto con le complesse forme di autogestione orizzontale e di distribuzione delle cariche interne a questi sodalizi ricreativi sempre più intrisi di spiriti di rivolta e nei quali pour cause «il confine tra la politica, il divertimento e la ribellione era labile e indeterminato» 8 .

Come modalità d'esecuzione, il canto collettivo stesso, suggellato dai cori all'unisono, sembrava essere quasi metafora delle esortazioni politiche all'unione fra pari ed implicava anzi la loro partecipazione e il loro protagonismo attivo (di cui Toss si spinge a sottolineare, a un certo punto, la vicinanza con le riflessioni poste alla base, ai giorni nostri, «degli Happening pop di delirio organizzato» e agli sforzi d'interazione col pubblico tentati nel Nuovo Teatro Popolare dal comico Paolo Rossi ${ }^{9}$ ). L'evoluzione delle goguettes, ad ogni modo, e in parallelo il moltiplicarsi degli esempi di politicizzazione offerti o proposti dai cantautori popolari quasi tutti di estrazione

7 BOISSON, Marius, Charles Gille ou le Chansonnier pendu (1820-1856). Histoire de la goguette, Paris, Peyronnet et Cie éditeurs, 1925, p. 57.

8 TOSS, Michele, op. cit., p. 124.

9 Cfr. ROSSI, Paolo, La commedia è finita! Conversazione delirante con Carolina De La Calle Casanova, Milano, Elèuthera, 2010. 
operaia e artigiana anche se non tutti guadagnati alla causa della lotta di classe, documentano nei dintorni del fatidico 1848 il consolidarsi di una inedita autonomia culturale dei ceti subalterni urbani sempre più inclini a reclamare l'instaurazione di un nuovo ordine repubblicano al tempo stesso democratico e sociale. Realisticamente fondato sulla esperienza diretta e materiale dello sfruttamento e di una quotidianità comunque marcata dalla durezza e dalla precarietà del lavoro, dalle discriminazioni $\mathrm{e}$ sovente dalla miseria, il canto si converte in invettiva contro le ingiustizie del capitalismo nascente $\mathrm{e}$ in affermazione orgogliosa di una identità popolare $\mathrm{e}$ antiborghese che definitivamente supera e radicalizza il senso dell'antica divisione tra poveri e ricchi, tra sottomessi e potenti.

Le goguettes, nonostante la finale sconfitta proletaria "sul campo", nel 1848, di molti dei loro frequentatori, proseguiranno a Parigi il proprio cammino arricchito dalla individuazione di obiettivi sempre più ambiziosi e manterranno viva sino alla fine del secolo XIX, passando anche attraverso i mesi di passione della Comune, una propria funzione sociale in grado, com'è stato spiegato da Axel Körner e da Hélène Millot ${ }^{10}$, di preservare, di quell'evento e dei valori che lo avevano animato, assieme al ricordo (remembrance) e alla memoria (remémoration), lo slancio utopico, l'orizzonte sociale e alla fine un progetto politico. Sul versante popolare, ossia dal punto di vista del "popolo" inteso come insieme delle classi subalterne, una tale eredità, di nuovo declinata in canti e suoni, avrebbe costituito, quanto meno in parte, l'equivalente di ciò che nella cultura borghese europea rappresentava già dai primi decenni dell'Ottocento, nell'età del melodramma e degli inni insurrezionali per l'indipendenza dei "popoli" d'Europa e del mondo, un nesso inequivocabile fra musica e politica di cui, come sappiamo e com'è stato più volte ribadito di recente (da Lorenzo Santoro, da Carlotta Sorba ecc. ${ }^{11}$ ) grande fu anche per l'Italia (non solo mazziniana), la rilevanza.

Il lascito degli chansonniers quarante-huitards, per come si delineò in termini creativi o anche di rielaborazione dell'eredità rivoluzionaria francese, tanto patriottica quanto giacobina nel rapporto tormentato ma evidente con il passato nazionale ${ }^{12}$, non a

${ }^{10}$ KÖRNER, Axel, Ideas and Memories of 1848 in France: Nationalism, République Universelle and Internationalism in the Goguette between 1848 and 1890, in ID. (ed. by), 1848: a European revolution? International Ideas and National Memories of 1848, London-New York, Palgrave Macmillan, 2000, pp. 85-105; MILLOT, Hélène, Légitimité et illégitimité de la voix du peuple: Charles Gille et la production chansonnière des goguettes de 1848, in MILLOT, Hélène, SAMINADAYER-PERRIN, Corinne (sous la direction de), 1848. Une révolution du discours, Saint-Etiernne, Ed. des Cahiers intempestifs, 2001, pp. 107-124.

${ }^{11}$ Cfr. SANTORO, Lorenzo, Musica e politica nell'Italia unita. Dall'illuminismo alla repubblica dei partiti, Venezia, Marsilio, 2013; SORBA, Carlotta, Il melodramma della nazione. Politica e sentimenti nell'età del Risorgimento, Roma-Bari, Laterza, 2015.

${ }^{12}$ AGULHON, Maurice, Les Quarante-huitards, Paris, Gallimard, 1992. 
caso dischiudeva infatti precise prospettive internazionaliste che tuttavia, fra battute d'arresto e contraddizioni vistose (a cominciare da quelle innescate nel 1849 dalla drammatica fine della Repubblica Romana), continuarono sempre ad ispirarsi a una precisa logica di rivendicazione sociale e a nuove e potenti immagini, come quella del peuple-roi, ch'erano state sbozzate a buon punto per propagandarle: «Le peuple est roi! La force est dans ses mains [...]/Tout vient du peuple, et tout doit être à lui!!».

Enunciata con strofe e melodie che costituivano qualcosa di più e di diverso d'una semplice espressione letteraria, era anche questa l'affermazione di un canone rivoluzionario, insieme politico e canoro, la cui portata sovversiva sarebbe rimbalzata più volte dalle barricate del ' 48 sino alle soglie del Novecento: «La liberté fera le tour du monde;/Le peuple est roi, monarques, chapeau bas!». 


\section{* L'autore}

Emilio Franzina è ordinario di Storia Contemporanea nell'Università di Verona presso Il Dipartimento TeSIS. I suoi interessi e i suoi studi spaziano dalle ideologie economiche allo sviluppo capitalistico e industriale, dalla diaristica alla scrittura popolare, anche se il suo campo d'indagine privilegiato, da circa quarant'anni, sono i movimenti migratori di massa. Tra i suoi numerosi libri si segnalano: La grande emigrazione. L'esodo dei rurali dal Veneto (Venezia, Marsilio 1976, tr. portoghese A grande emigração, Campinas, Unicamp, 2006); Merica! Merica! Emigrazione e colonizzazione nelle lettere dei contadini veneti e friulani in America latina 1876-1902 (Milano, Feltrinelli, 1979; Verona, Cierre Editori 2001); Storia dell'emigrazione veneta dall'unità al fascismo (Verona, Cierre, 1991); Gli italiani al nuovo mondo. L'emigrazione italiana in America 1492-1942 (Milano, Mondadori 1995); Storia dell'emigrazione italiana (dir.), 2 voll.(Roma, Donzelli, 2001-2002); Una patria espatriata. Lealtà nazionale e caratteri regionali nell'immigrazione italiana all'estero (secoli XIX e XX), (Viterbo, Settecittà, 2006); L'America gringa. Storie italiane d'immigrazione tra Argentina e Brasile (Reggio Emilia, Diabasis, 2008); La terra ritrovata. Storiografia e memoria della prima immigrazione italiana in Brasile (Genova, Termanini, 2014); La storia (quasi vera) del Milite ignoto raccontata come un'autobiografia (Roma, Donzelli, 2014). Collabora, sedendo anche nel loro comitato scientifico, con varie riviste specializzate come "Altreitalie», «Studi Emigrazione», «Estudios Migratorios Latinoamericanos» e, dopo averlo fondato nel 2004, dirige con Matteo Sanfilippo l'Archivio storico dell'emigrazione italiana.

URL: < http://www.studistorici.com/progett/autori/\#Franzina >

\section{Per citare questo articolo:}

FRANZINA, Emilio, «Recensione: Michele TOSS, Il Popolo Re. La canzone sociale a Parigi (1830-1848), Bologna, Clueb, 2014, 218 pp.», Diacronie. Studi di Storia Contemporanea : Costruire. Rappresentazioni, relazioni, comunità, 29/06/2015,

URL:< http://www.studistorici.com/2015/06/29/franzina_numero_22/ >

Diacronie Studi di Storia Contemporanea $\beta$ www.diacronie.it

Risorsa digitale indipendente a carattere storiografico. Uscita trimestrale. redazione.diacronie@hotmail.it

Comitato di redazione: Jacopo Bassi - Luca Bufarale - Elisa Grandi - Deborah Paci - Fausto Pietrancosta - Matteo Tomasoni - Luca Zuccolo

Diritti: gli articoli di Diacronie. Studi di Storia Contemporanea sono pubblicati sotto licenza Creative Commons 2.5 Possono essere riprodotti a patto di non modificarne i contenuti e di non usarli per fini commerciali. La citazione di estratti è comunque sempre autorizzata, nei limiti previsti dalla legge. 